\title{
Control Mechanisms of Photoisomerization in Protonated Schiff Bases
}

Lela Vuković, ${ }^{*}+\dagger$ Carl F. Burmeister, ${ }^{\dagger}$ Petr Král, $^{\ddagger, \S}$ and Gerrit Groenhof* ${ }^{*}, \dagger$

${ }^{\dagger}$ Department of Theoretical and Computational Biophysics, Max Planck Institute for Biophysical Chemistry, Am Fassberg 11, D-37077 Göttingen, Germany

${ }^{\ddagger}$ Department of Chemistry and ${ }^{\S}$ Department of Physics, University of Illinois at Chicago, Chicago, Illinois 60607, United States

"Department of Chemistry and Nanoscience Center, University of Jyväskylä, P.O. Box 35 FI-40014 Jyväskylä, Finland

\section{Supporting Information}

ABSTRACT: We performed ab initio excited-state molecular dynamics simulations of a gas-phase photoexcited protonated Schiff base $\left(C_{1}-N_{2}=C_{3}-C_{4}=C_{5}-C_{6}\right)$ to search for control mechanisms of its photoisomerization. The excited molecule twists by $\sim 90^{\circ}$ around either the $\mathrm{N}_{2} \mathrm{C}_{3}$ bond or the $\mathrm{C}_{4} \mathrm{C}_{5}$ bond and relaxes to the ground electronic state through a conical intersection with either a trans or cis outcome. We show that a large initial distortion of several dihedral angles and a specific normal vibrational mode combining pyramidalization and double-bond twisting can lead to a preferential rotation of atoms around the $\mathrm{C}_{4} \mathrm{C}_{5}$ bond. We also show that selective pretwisting of several dihedral angles in the initial ground state thermal ensemble (by analogy to a protein pocket) can significantly increase the fraction of photoreactive (cis $\rightarrow$ trans) trajectories. We demonstrate that new ensembles with higher degrees of control over the photoisomerization reaction can be obtained by a computational directed evolution approach on the ensembles of molecules with the pretwisted geometries.

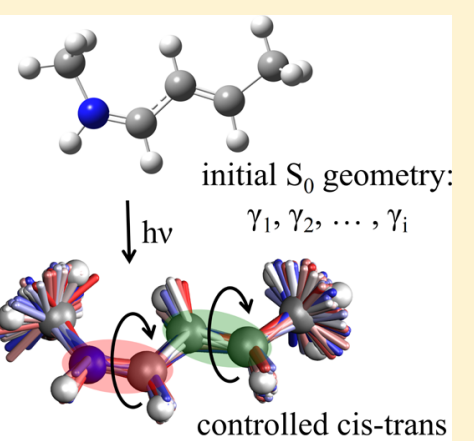

conversion on $\mathrm{S}_{1}$

SECTION: Spectroscopy, Photochemistry, and Excited States

U ltrafast cis-trans photoisomerization of double bonds in small photoactive molecules is a first step in many lightinduced biological processes. ${ }^{1}$ It can also be used in lightpowered molecular and nanoscale devices. ${ }^{2,3}$ Many biological photoisomerization reactions involve the protonated Schiff base (PSB) chromophore retinal, which when photoexcited in its native protein environment leads to vision, light-induced opening of channel proteins, and other biological phenomena. $^{4-6}$ Typically, retinal on the ground potential energy surface (PES), $\mathrm{S}_{0}$, absorbs light, relaxes, and reaches a conical intersection (CI) on the first excited PES, $S_{1}$. Then, it passes through the CI back to the $S_{0}$ PES with the initial or changed (trans, cis) minimum conformation. ${ }^{7-12}$

Photoisomerization was studied in different PSBs and retinal, located in vacuum, in the presence of counterions, different solvents, and proteins. ${ }^{13-22}$ It was shown that numerous factors can influence the outcome of photoisomerization reactions, such as the length of the conjugate chain in the PSB chromophore, ${ }^{19}$ steric restraints (helicity) imposed on the chromophore by the protein environment, ${ }^{19,23}$ the topography of the $\mathrm{CI},{ }^{24}$ and the phase of the vibration of the torsional coordinates coupled to the reactive coordinate. ${ }^{10,25,26}$ Moreover, the isomerization outcome (yield) for retinal in the native protein environment can be coherently controlled under weakfield conditions. ${ }^{27}$ However, to the best of our knowledge, the impact of the initial geometry and vibrational excitation on the reaction outcome of the PSB photoisomerization has not been systematically studied.
We actively search for mechanisms that may be used to control the photoisomerization outcome of a small photoexcited PSB, such as the bond selectivity and (trans, cis) conformation. We use complete active space self-consistent field (CASSCF) description and ab initio molecular dynamics to simulate a gas-phase photoexcited PSB with two double bonds (PSB2), as described in the Methods (Supporting Information (SI)). Note that PSB2 photoisomerization takes place on the ionic $\left(1 \mathrm{~B}_{2}\right) S_{1}$ state $^{28}$ as in retinal but does not feature a "central" trans double bond flanked by a polyene and a polyeneiminium.

Relaxation Dynamics of the Photoexcited Thermal Ensemble. In Chart 1, we show the PSB and its two main relaxation paths on the excited state $\left(S_{1}\right)$ electronic PES upon photoexcitation. The relaxation on $S_{1}$ has two dominant features: (1) "Bond order inversion" occurs, where $\mathrm{N}_{2} \mathrm{C}_{3}, \mathrm{C}_{4} \mathrm{C}_{5}$ bonds stretch and gain "single-bond" character while the $\mathrm{C}_{3} \mathrm{C}_{4}$ bond shortens and gains "double-bond" character. (2) Twisting by $\sim 90^{\circ}$ occurs around either the $\mathrm{N}_{2} \mathrm{C}_{3}$ bond or the $\mathrm{C}_{4} \mathrm{C}_{5}$ bond. Through this $S_{1}$ relaxation, the PSB2 reaches a CI seam region corresponding to one of the two isomerizing double bonds (twisting to $\sim 90^{\circ 29}$ ). At the seam, the molecule hops to $S_{0} P E S$, as described here by two hopping algorithms, diabatic surface

Received: January 16, 2013

Accepted: March 6, 2013

Published: March 6, 2013 
hopping $(\mathrm{DSH})^{30}$ and fewest switches hopping $(\mathrm{FSH})^{31}$ algorithms. The $S_{1}$ relaxation of PSB2 is similar to that taken by the (full) retinal Schiff base. ${ }^{11}$ Whereas we describe the PSB2 molecule at the $\operatorname{CASSCF}(4,4) / 6-31 G^{*}$ level of theory (SI), our xMCQDPT2/CASSCF $(4,4) /$ cc-pVTZ calculations $^{32}$ (SI, Figures 2 and 3 ) and recent studies have shown that dynamic electron correlation can modify the shape of the $S_{1}$ PES energy, ${ }^{33}$ and thus affect the excited-state relaxation. We use two surface-hopping algorithms to confirm that the observed correlations between the reaction outcome distributions and the initial molecular geometries are not dependent on the hopping method. However, because hops are restricted to the seam in the DSH algorithm, the excited-state lifetime is significantly longer if the DSH algorithm is used, in line with previous observations. 30

In Table 1, we summarize the reaction outcome statistics and the excited-state decay times, $\tau_{\mathrm{DSH}}$ and $\tau_{\mathrm{FSH}}$, obtained for 296

\section{Chart 1. Protonated Schiff Base ${ }^{a}$}

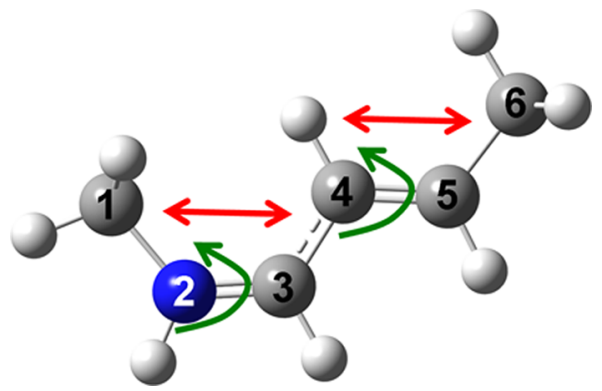

${ }^{a}$ Arrows indicate two main relaxation paths upon photoexcitation: (red arrows) bond order inversion, where the $\mathrm{N}_{2} \mathrm{C}_{3}$ and $\mathrm{C}_{4} \mathrm{C}_{5}$ bonds stretch and $\mathrm{C}_{3} \mathrm{C}_{4}$ shortens; (green arrows) twisting around either the $\mathrm{N}_{2} \mathrm{C}_{3}$ or $\mathrm{C}_{4} \mathrm{C}_{5}$ bonds.

Table 1. $S_{1} \rightarrow S_{0}$ Reaction Outcome Statistics, Including the Excited State Decay Time $\left(S_{1}\right.$ population fitted to $\left.e^{-t / \tau}\right)$, the Number of Occurrences of the Specified Reaction Outcome $i$, and the Related Percentages

\begin{tabular}{lllll} 
outcome & $\mathrm{N}_{2} \mathrm{C}_{3}$ cis & $\mathrm{N}_{2} \mathrm{C}_{3}$ trans & $\mathrm{C}_{4} \mathrm{C}_{5}$ cis & $\mathrm{C}_{4} \mathrm{C}_{5}$ trans \\
$\tau_{\mathrm{DSH}}[\mathrm{fs}]$ & $96 \pm 1$ & $132 \pm 2$ & $49 \pm 1$ & $51 \pm 1$ \\
$\mathrm{~N}_{i, \mathrm{DSH}}$ & 132 & 105 & 32 & 27 \\
$\mathrm{P}_{i, \mathrm{DSH}}[\%]$ & 44.6 & 35.5 & 10.8 & 9.1 \\
$\tau_{\mathrm{FSH}}[\mathrm{fs}]$ & $65 \pm 1$ & $74 \pm 1$ & $42 \pm 1$ & $50 \pm 1$ \\
$\mathrm{~N}_{i, \mathrm{FSH}}$ & 209 & 172 & 67 & 46 \\
$\mathrm{P}_{i, \mathrm{FSH}}[\%]$ & 42.3 & 34.8 & 13.6 & 9.3 \\
\hline
\end{tabular}

and 494 trajectories in which the DSH and the FSH algorithms were used, ${ }^{30,31}$ respectively. The reaction outcome is classified according to which double bond $\left(\mathrm{N}_{2} \mathrm{C}_{3}\right.$ or $\left.\mathrm{C}_{4} \mathrm{C}_{5}\right)$ twists upon the initial photoexcitation, and according to the minimum (trans or cis) in which the molecule ends up in on the $S_{0}$ PES after the hop. We observe that the $\mathrm{N}_{2} \mathrm{C}_{3}$ bond (initially cis) twists in $\sim 80 \%$ of the cases (44.6 (42.4) cis and 35.5 (34.8) trans outcome for DSH (FSH) algorithms), whereas the $\mathrm{C}_{4} \mathrm{C}_{5}$ (initially trans) twisting takes place in the remaining $\sim 20 \%$ trajectories. Our simulations indicate that no other relaxation pathways are accessible to the molecule. The two observed photoisomerization paths lead to different excited-state decay times, with the $\mathrm{N}_{2} \mathrm{C}_{3}$ bond isomerization on $\mathrm{S}_{1}$ PES occurring within $\tau_{\mathrm{DSH}} \approx 96-132$ fs or $\tau_{\mathrm{FSH}} \approx 65-74$ fs and the $\mathrm{C}_{4} \mathrm{C}_{5}$ bond isomerization occurring within $\tau_{\mathrm{DSH}} \approx 49-51$ fs or $\tau_{\mathrm{FSH}} \approx$
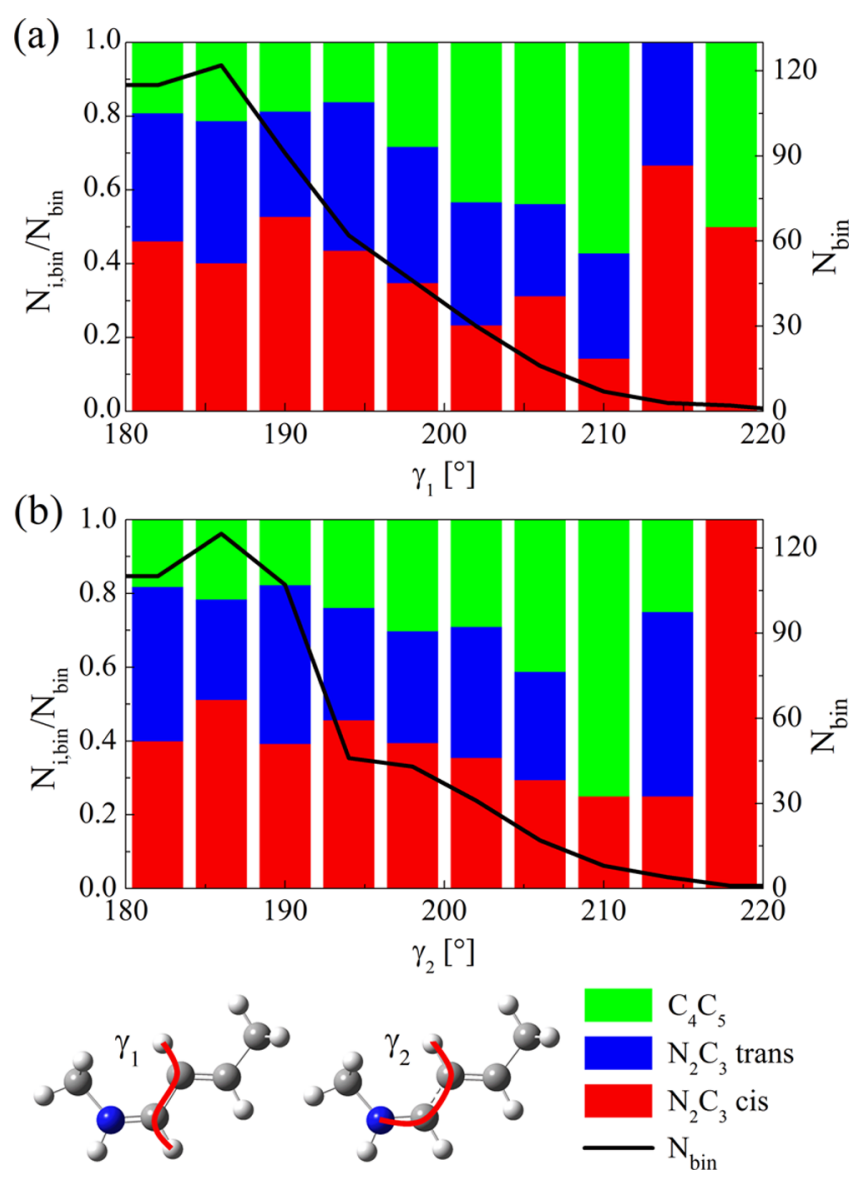

Figure 1. (a,b) Black lines (right axis) show the distributions of the $\gamma_{j}$ dihedral angles (symmetrized) in the PSB2 molecules in the initial thermal ensemble. (Integration of the black lines gives the total number of geometries/trajectories.) The histograms show the relative values of the different possible photoreaction outcomes, $N_{\mathrm{i}, \text { bin }} / N_{\text {bin }}$, for each value of the initial dihedral. The data are from the thermal ensemble trajectories obtained with the FSH algorithm. ${ }^{31}$ The procedures used to generate the thermal ensemble are described in the SI.

42-50 fs, in dependence of the hopping algorithm. The different excited-state lifetimes obtained by the use of the two hopping algorithms originate in the fact that the hopping occurs in the DSH method only when the molecule is crossing the CI seam, whereas in the FSH method the hopping is allowed prior to the molecule reaching the seam, as discussed by Fabiano et al. ${ }^{30}$

To determine why the $\mathrm{N}_{2} \mathrm{C}_{3}$ isomerization is favored, we examine the slope of the $S_{1}$ PES in the regions visited by the molecule immediately after the vertical photoexcitation. Because the two main relaxation pathways of the molecule involve torsion of either the $\mathrm{CN}_{2} \mathrm{C}_{3} \mathrm{C}$ or $\mathrm{CC}_{4} \mathrm{C}_{5} \mathrm{C}$ dihedral angles, we perform rigid scans of the $\mathrm{CN}_{2} \mathrm{C}_{3} \mathrm{C}$ and $\mathrm{CC}_{4} \mathrm{C}_{5} \mathrm{C}$ dihedral angle $(\gamma)$ changes on the $S_{1}$ PES. When doing so, we keep either the original $S_{0}$-like bond lengths (typically preserved in the initial stage of the $S_{1}$ PES dynamics) or the inverted $S_{1}$-like bond lengths (typically appearing within $\sim 5-$ $10 \mathrm{fs}$ upon the vertical photoexcitation). The dihedral change scans in these two cases show that when the molecule keeps its initial $\mathrm{S}_{0}$-like nuclear positions (bond lengths) the energy profile for the $\mathrm{CC}_{4} \mathrm{C}_{5} \mathrm{C}$ dihedral change is much steeper than the profile for the $\mathrm{CN}_{2} \mathrm{C}_{3} \mathrm{C}$ change (SI, Figure 2). In contrast, after the nuclei (bond lengths) in the molecule adjust to the $S_{1}$ 


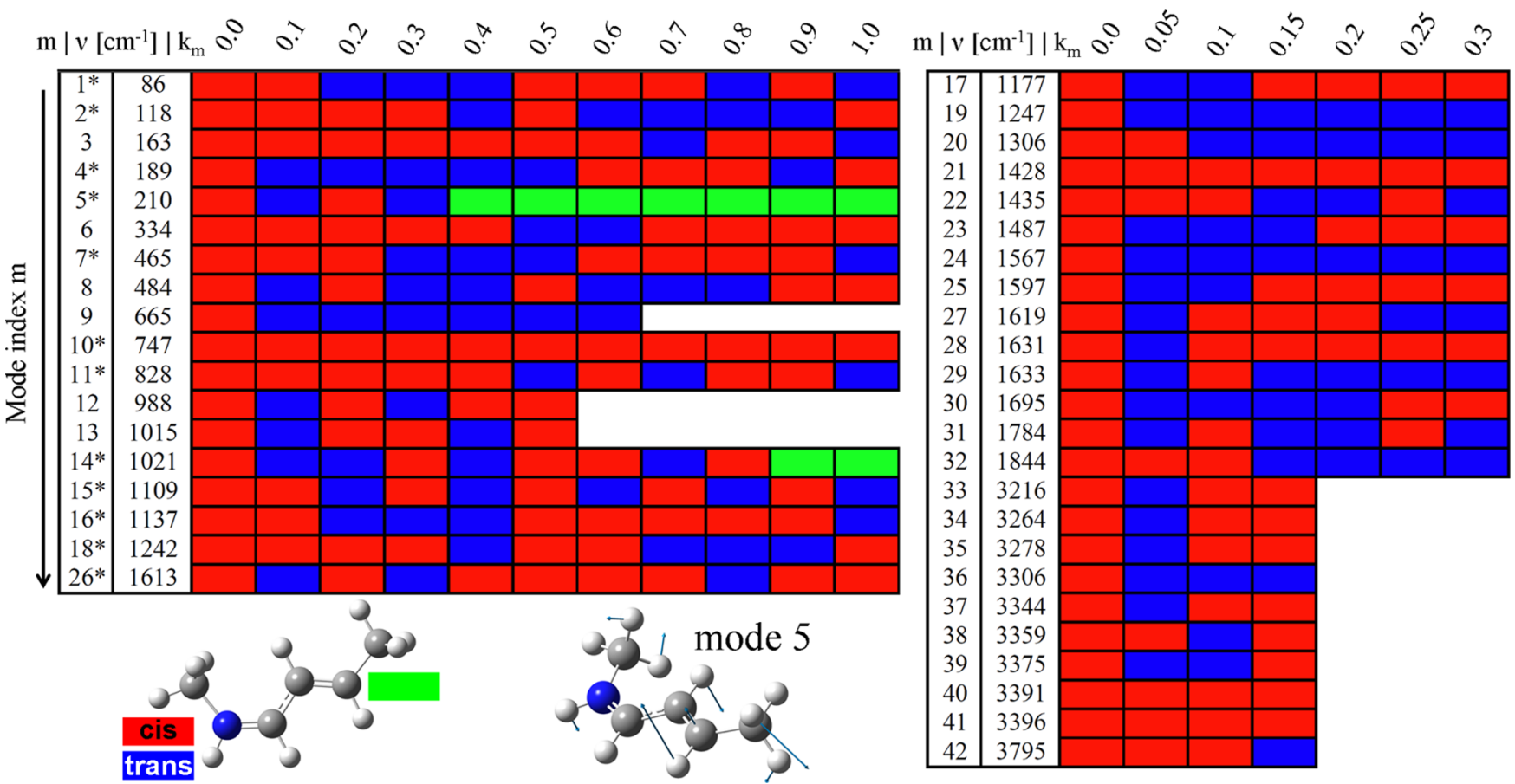

Figure 2. Table of reaction outcomes obtained from the dynamics trajectories of photoexcited PSB2 with initial geometries of the cold molecule preexcited along individual normal modes. The label $(*)$ indicates modes with "out-of-plane displacements" of their atoms (text). (inset) Three possible reaction outcomes are marked schematically (isomerization of $\mathrm{N}_{2} \mathrm{C}_{3}$ bond with trans and cis outcomes and isomerization of $\mathrm{C}_{4} \mathrm{C}_{5}$ bond with any outcome). Mode 5 (in the PSB2), whose pre-excitation leads to $\mathrm{C}_{4} \mathrm{C}_{5}$ isomerization at $k_{m}>0.3$, has prominent displacements of hydrogen atoms bound to $\mathrm{C}_{4}$ and $\mathrm{C}_{5}$ atoms (animation of atomic displacements along the mode 5 is included in the $\mathrm{SI}$ ). In these trajectories, we used the DSH algorithm. $^{30}$

PES, the energy profile for the $\mathrm{CN}_{2} \mathrm{C}_{3} \mathrm{C}$ change becomes steeper (SI, Figure 2). This is likely related to the above observed higher probability $(80 \%)$ of the $\mathrm{N}_{2} \mathrm{C}_{3}$ isomerization pathway. The large probability can be understood from the fact that the rotation frequency around the $\mathrm{C}_{4} \mathrm{C}_{5}$ bond should be much smaller than the vibration frequency associated with the bond length rearrangement on the $S_{1}$ PES, which means that the $\mathrm{N}_{2} \mathrm{C}_{3}$ channel becomes much more likely. However, certain initial molecular geometries or nuclear motions may change this trend upon the photoexcitation.

Control of Bond Selectivity. It is important to understand how the quantum yield and the bond selectivity can be affected by the initial geometry of the photoexcited chromophore. The deformation/pretwisting of the PSB retinal and variation of its normal modes within a protein pocket may determine the (enhanced) quantum yield and bond selectivity observed in nature. ${ }^{16}$ To pursue this idea, we explore on a large set of initial PSB2 geometries if certain distortions can significantly influence the photoreaction pathways and outcomes. We expand the initial molecular geometries (and velocities) of the PSB2 thermal ensemble in terms of the internal molecular coordinates (bond lengths, torsional angles). Alternatively, we project these geometries on the vibrational normal modes (discussed later) to see if any of them significantly affect the outcome. The obtained knowledge may clarify how natural chromophores operate, and it can also be useful for designing photoactive molecular devices.

In Figure 1a,b, we show the histograms with relative values of the different possible photoreaction outcomes, $N_{i, \text { bin }} / N_{\text {bin }}$, for each value of the initial dihedral $\gamma_{j}\left(N_{\text {bin }}=\sum_{i} N_{i, \text { bin }}\right.$, where $i$ is the reaction outcome and $N_{i, \text { bin }}$ is the number of trajectories within a single bin that have outcome $i$ ). The two dihedrals angles $\gamma_{j}$, surrounding the $\mathrm{C}_{3} \mathrm{C}_{4}$ bond, are varied in $4^{\circ}$-wide bins. As the dihedrals increase from $\gamma_{j} \approx 180$ to $195-210^{\circ}$, the probability that the reaction occurs along the $\mathrm{C}_{4} \mathrm{C}_{5}$ pathway increases from 20 to $\sim 30-40 \%$. Therefore, the reaction outcome (bond selectivity) is sensitive to the molecular twisting in the region surrounding the $\mathrm{C}_{3} \mathrm{C}_{4}$ bond. Note that only a few cases are observed at $\gamma_{j}>210^{\circ}$ and that the errors in the observed outcome distributions become larger as $\gamma_{j}$ increases. No significant effect on the reaction outcome was observed when other internal coordinates were considered.

Systematic Pre-excitation of Single Normal Modes. Next, we examine how the photoisomerization outcome is affected by the pre-excitation of individual vibrational normal modes of the molecule. We prepare a set of initial molecular structures, with the nuclear coordinates $\vec{R}_{m}$, by pre-exciting (distorting) a cold PSB2 molecule, with the nuclear coordinates $\vec{R}_{0}$, along single normal modes, with unit displacements $\vec{N}_{m}$ and excitation amplitudes $k_{m}$

$$
\vec{R}_{m}=\vec{R}_{0}+k_{m} \vec{N}_{m}
$$

The $\vec{R}_{0}$ coordinates correspond to the molecular geometry at the $S_{0}$ minimum, calculated at the $\operatorname{CASSCF}(4,4) / 6-31 G^{*}$ level of theory. Because some of the normal modes represent rotational motions (curvilinear motion in Cartesian space), we express $\vec{N}_{m}$ in the nonredundant internal coordinates (described in the SI).

In Figure 2, we show the photoisomerization outcomes for the cold PSB2 molecule pre-excited along its 42 normal modes. In 12 of these modes, 10 atoms $(5 \mathrm{C}, 1 \mathrm{~N}, 4 \mathrm{H})$ forming the planar conjugated region show out-of-plane displacements, while in 30 modes the frame remains planar. Several lowfrequency out-of-plane modes correspond to anti-pyramidaliza- 
tion ("propelling" displacement) of hydrogens around double bonds (e.g., hydrogens motion around the $\mathrm{C}_{4} \mathrm{C}_{5}$ bond in mode 5 , shown in the inset of Figure 2) or syn-pyramidalization (displacement in the same direction) of those hydrogens (e.g., the same atoms in modes 11 and 15). ${ }^{34}$ Upon photoexcitation of PSB2 to the $S_{1}$ PES, the pre-excitation of almost all of the modes leads to isomerization of the $\mathrm{N}_{2} \mathrm{C}_{3}$ double bond, whereas isomerization of the $\mathrm{C}_{4} \mathrm{C}_{5}$ bond is observed only if the modes 5 and 14 are pre-excited. At room temperature, only the mode 5 would be excited to $k_{m}>0.3$, leading to the $\mathrm{C}_{4} \mathrm{C}_{5}$ bond isomerization (Figure 2 in the SI). Interestingly, excitation of the mode 5 leads to a displacement of the $\mathrm{C}_{4}$-bound hydrogen atom and simultaneous change of the $\gamma_{1}$ and $\gamma_{2}$ dihedrals (Figure 1). Therefore, pretwisting of the $\gamma_{1}$ and $\gamma_{2}$ dihedrals in mode 5 might lead to the bond selectivity in the PSB2 photoisomerization reaction.

In Figure $3 \mathrm{a}$, we present the time evolution of the $\mathrm{HC}_{4} \mathrm{C}_{5} \mathrm{H}$ dihedral on the $S_{1}$ PES for PSB2 initially pre-excited $\left(k_{m}\right)$ along the mode 5 (prior to vertical $S_{0} \rightarrow S_{1}$ photoexcitation). At $k_{m}<$ 0.375 , only the $\mathrm{N}_{2} \mathrm{C}_{3}$ bond is activated, while pretwisting of the mode 5 with $k_{m} \geq 0.375$ leads to activation of the $\mathrm{C}_{4} \mathrm{C}_{5}$ bond. As $k_{m}$ increases, change of the $\mathrm{HC}_{4} \mathrm{C}_{5} \mathrm{H}$ dihedral and eventual hopping to the $S_{0}$ PES occurs faster. (The hopping times are 77 and 43 fs for $k_{m}=0.4$ and 1.0, respectively.) Figure $3 \mathrm{a}$ also shows that as $k_{m}$ grows the outcome of the photoisomerization around the $\mathrm{C}_{4} \mathrm{C}_{5}$ bond starts to oscillate between the (trans, cis) forms.

Normal Mode Analysis of a Thermal Ensemble. We now examine the modal composition in the initial PSB2 configurations of the thermal ensemble (also analyzed in Figure 1) to find out how it influences the photoisomerization outcome. We extract the modal composition by the leastsquares fitting procedure, where we project each initial configuration $\vec{R}$ on the "cold" $\left(S_{0}\right)$ configuration $\vec{R}_{0}$ with added displacements of the normal modes $k_{m} \vec{N}_{m}$

$$
d=\left|\vec{R}-\vec{R}_{0}-\sum_{m=1}^{42} k_{m} \vec{N}\right|^{2}
$$

As noted before, all of the vectors are expressed on the basis of nonredundant internal coordinates.

In Figure $3 b$, we show histograms with the relative values of the different possible photoreaction outcomes, $N_{\mathrm{i}, \text { bin }} / N_{\text {bin }}$, for each value of the mode 5 coefficient $k_{m=5}$. We can see that in the thermal ensemble a higher population of the mode 5 also leads to an increased probability of the $\mathrm{C}_{4} \mathrm{C}_{5}$ bond isomerization. At $k_{5}<0.4$, the probability of isomerization is $\sim 20 \%$, but for $k_{5}=0.4-0.7$, it increases to $30-50 \%$. (Only a few initial geometries have $k_{5}>0.7$.)

Control of the (trans, cis) Outcome. Despite the observed correlations between the initial molecular geometry and the isomerizing bond, we did not find any correlations between this geometry and the (trans, cis) reaction outcome $\left(\mathrm{N}_{2} \mathrm{C}_{3}\right.$ bond analyzed). However, these correlations emerged when the velocities along the internal coordinates were also considered.

In Figure 3c, we plot the angular velocities for the $\mathrm{HN}_{2} \mathrm{C}_{3} \mathrm{H}$ (hydrogen) and $\mathrm{CN}_{2} \mathrm{C}_{3} \mathrm{C}$ (carbon) dihedrals at the point of hopping, sorted according to the reaction outcome. We plot these results for the DSH (left) and FSH (right) algorithms and limit our analysis to the cases in which the $\mathrm{N}_{2} \mathrm{C}_{3}$ bond is isomerized ( $80 \%$ of all cases). These results reveal that the (trans, cis) outcome is determined by the sign of the angular velocity of the $\mathrm{HN}_{2} \mathrm{C}_{3} \mathrm{H}$ torsional angle at the point of hopping
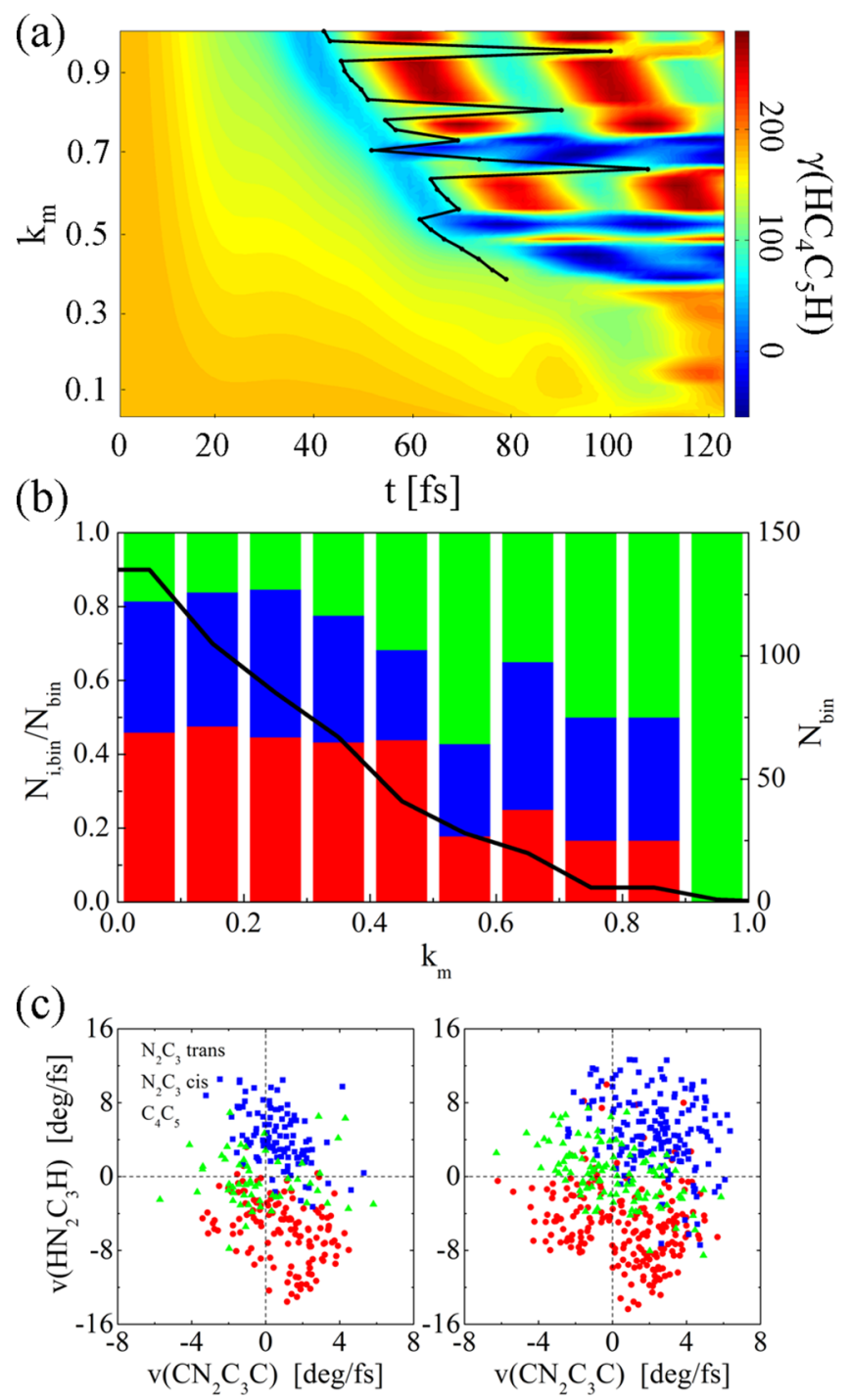

Figure 3. (a) Time evolution of the $\mathrm{HC}_{4} \mathrm{C}_{5} \mathrm{H}$ dihedral upon the $\mathrm{S}_{0} \rightarrow$ $S_{1}$ photoexcitation of PSB2 for initial geometries pre-excited along the normal mode 5 ( $k_{m=5}$, probed in 0.025 -wide increments). At $k_{5}>0.35$, the $\mathrm{C}_{4} \mathrm{C}_{5}$ bond isomerization becomes activated, as seen in the dihedral evolution. After reaching the $\mathrm{CI}$, corresponding to geometries with the twisted $\mathrm{HC}_{4} \mathrm{C}_{5} \mathrm{H}$ and $\mathrm{CC}_{4} \mathrm{C}_{5} \mathrm{C}$ dihedrals, the molecule eventually hops to the cis outcome (dark blue) or trans outcome (red) at the $\mathrm{C}_{4} \mathrm{C}_{5}$ bond. The black line marks the hopping times for all of the trajectories (DSH algorithm used). (b) Black line (right axis) shows the distribution of the $k_{5}$ values in the PSB2 molecules in the initial thermal ensemble. The histograms show the relative values of the different possible photoreaction outcomes, $N_{i, \text { bin }} / N_{\text {bin }}$, for each value of the $k_{5}$ (symmetrized). The data are from the thermal ensemble trajectories analyzed in Figure 1 (FSH algorithm). Legend is the same as in Figure 1. (c) Angular velocities for the $\mathrm{HN}_{2} \mathrm{C}_{3} \mathrm{H}$ and $\mathrm{CN}_{2} \mathrm{C}_{3} \mathrm{C}$ dihedrals at the hopping point for all the trajectories, sorted according to the outcome. (left) DSH and (right) FSH algorithms.

from $S_{1}$ to $S_{0}$ PES, as in other PSBs ${ }^{10,35-37}$ and analogs of the photoactive yellow protein chromophore. ${ }^{38}$ However, if the wave function remains $S_{1}$-like immediately after the hopping to the $S_{0}$ PES, then the cis or trans outcome may be different than predicted by the velocity along the $\mathrm{HN}_{2} \mathrm{C}_{3} \mathrm{H}$ torsional angle. ${ }^{37}$

The correlation of the hydrogen-dihedral velocity with the (trans, cis) outcome can be explained by the fact that the direction of the (fast) relative motion of the two hydrogens 

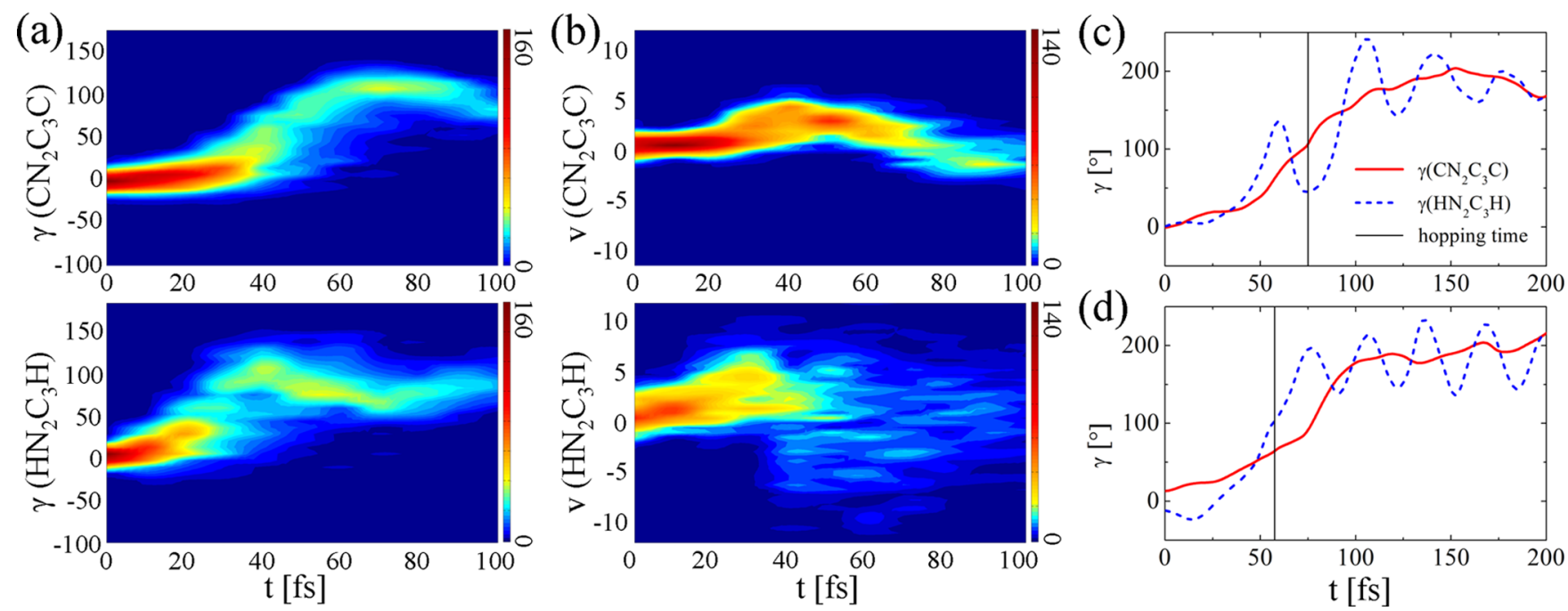

Figure 4. Time-dependent distributions of (a) the $\mathrm{CN}_{2} \mathrm{C}_{3} \mathrm{C}$ and $\mathrm{HN}_{2} \mathrm{C}_{3} \mathrm{H}$ dihedrals (absolute values) and (b) their angular velocities obtained from the thermal ensemble trajectories in which the dihedrals around the $\mathrm{N}_{2} \mathrm{C}_{3}$ bond change on the $\mathrm{S}_{1}$ PES, prior to hopping to the $\mathrm{S}_{0}$ (FSH). (c) Timedependent evolution of the $\mathrm{CN}_{2} \mathrm{C}_{3} \mathrm{C}$ and $\mathrm{HN}_{2} \mathrm{C}_{3} \mathrm{H}$ dihedrals for a representative trajectory leading to trans outcome around the $\mathrm{N}_{2} \mathrm{C}_{3}$ bond. (d) Same for a representative trajectory in which $\mathrm{CN}_{2} \mathrm{C}_{3} \mathrm{C}$ and $\mathrm{HN}_{2} \mathrm{C}_{3} \mathrm{H}$ evolve synchronously.

Table 2. Fractions of Synchronous Trajectories, $P_{\text {sync }}$, Obtained in Different Thermal Ground State Ensembles, Calculated by DSH and FSH Algorithms

\begin{tabular}{|c|c|c|c|c|c|c|}
\hline therm. ens. & $\gamma_{1}\left[{ }^{0}\right]^{a}$ & $\gamma_{2}\left[^{\circ}\right]$ & $\gamma_{3}\left[^{\circ}\right]$ & $\gamma_{4}\left[^{\circ}\right]$ & $P_{\text {sync }}$ & $N_{\text {traj }}$ \\
\hline unconstr. FSH & & & & & $7.63 \%$ & 494 \\
\hline unconstr. DSH & & & & & $1.0 \%$ & 296 \\
\hline $1 \mathrm{FSH}, \gamma_{i}{ }^{16}$ & -17.5 & 168.6 & 174.1 & & $6.06 \%$ & 99 \\
\hline $2 \mathrm{FSH}$ & 13.0 & 150.0 & -172.4 & & $18.09 \%$ & 187 \\
\hline $2 \mathrm{DSH}$ & 13.0 & 150.0 & -172.4 & & $16.24 \%$ & 193 \\
\hline $3 \mathrm{FSH}$ & -20.63 & -154.4 & 172.9 & & $14.57 \%$ & 199 \\
\hline 3b FSH & -20.63 & -154.4 & 172.9 & 11.1 & $14.21 \%$ & 197 \\
\hline
\end{tabular}

${ }^{a} \gamma_{1-4}$ represents $\mathrm{C}_{1} \mathrm{~N}_{2} \mathrm{C}_{3} \mathrm{C}_{4}, \mathrm{~N}_{2} \mathrm{C}_{3} \mathrm{C}_{4} \mathrm{C}_{5}, \mathrm{C}_{3} \mathrm{C}_{4} \mathrm{C}_{5} \mathrm{C}_{6}$, and $\mathrm{HN}_{2} \mathrm{C}_{3} \mathrm{H}$, respectively. These angles are either free (marked by -) or (harmonically) restrained to the listed values.

attached to the $\mathrm{N}_{2}$ and $\mathrm{C}_{3}$ atoms determines the direction in which the $\mathrm{p}$ orbitals (opposing the hydrogens) on those atoms overlap at the point of hopping and re-establish the double bond. ${ }^{0,35-37}$ Rebuilding of the double bond from these $p$ orbitals is thus mostly controlled by the hydrogens moving faster than the much heavier opposing carbons.

Pretwisting Control of the (trans, cis) Outcome. After taking a closer look at the relaxation dynamics along the $\mathrm{N}_{2} \mathrm{C}_{3}$ reaction path on the $S_{1}$ surface, we notice that the change of the $\mathrm{HN}_{2} \mathrm{C}_{3} \mathrm{H}$ dihedral mostly precedes the change of the $\mathrm{CN}_{2} \mathrm{C}_{3} \mathrm{C}$ dihedral; this was also observed in other PSBs. ${ }^{11,37,39}$ However, significant change of both dihedrals is necessary for reaching the CI. (In the optimized CI geometry, both dihedrals are $\sim 90^{\circ 29}$.) It is of interest to examine further how the dynamics of the two dihedrals guides the molecule to the CI.

In Figure $4 a, b$, we show the time-dependent probability distributions of the $\mathrm{CN}_{2} \mathrm{C}_{3} \mathrm{C}$ and $\mathrm{HN}_{2} \mathrm{C}_{3} \mathrm{H}$ dihedrals and their angular velocities, with hopping described by the FSH algorithm. The data are obtained for photoexcitation from the thermal ensemble, where only the cases with the $\mathrm{N}_{2} \mathrm{C}_{3}$ bond isomerization (prior to $\mathrm{S}_{0}$ PES hopping) are considered. We can see that $\mathrm{HN}_{2} \mathrm{C}_{3} \mathrm{H}$ reaches the twisted geometry $\left(\sim 90^{\circ}\right)$ prior to $\mathrm{CN}_{2} \mathrm{C}_{3} \mathrm{C}$. After $\mathrm{HN}_{2} \mathrm{C}_{3} \mathrm{H}$ twists, it starts oscillating around $90^{\circ}$, whereas $\mathrm{CN}_{2} \mathrm{C}_{3} \mathrm{C}$ still continues changing toward this angle. A sample of this dynamics is shown on a single trajectory in Figure 4c. Because the molecule can hop (reach
CI) when both the $\mathrm{CN}_{2} \mathrm{C}_{3} \mathrm{C}$ and $\mathrm{HN}_{2} \mathrm{C}_{3} \mathrm{H}$ dihedrals are changed out of plane, $\mathrm{CN}_{2} \mathrm{C}_{3} \mathrm{C}$ needs to catch up with $\mathrm{HN}_{2} \mathrm{C}_{3} \mathrm{H}$. This happens at $t \approx 40-60 \mathrm{fs}$, when the $\mathrm{HN}_{2} \mathrm{C}_{3} \mathrm{H}$ velocities are significantly randomized and partially negative. Interestingly, in some trajectories of the thermal ensemble ( $\sim 7.63 \%$ in the FSH algorithm, Table 2 ), the $\mathrm{CN}_{2} \mathrm{C}_{3} \mathrm{C}$ and $\mathrm{HN}_{2} \mathrm{C}_{3} \mathrm{H}$ dihedrals evolve in a relatively "synchronous" way, as seen in Figure 4d. In these synchronous trajectories, the hopping to the $\mathrm{S}_{0}$ PES occurs before $\mathrm{HN}_{2} \mathrm{C}_{3} \mathrm{H}\left(\mathrm{CN}_{2} \mathrm{C}_{3} \mathrm{C}\right)$ reverses the rotation direction.

Our observation that the hydrogen dihedral change precedes the carbon change is in contrast with the observations made in longer PSBs. Previous studies ${ }^{11,16,37}$ have shown that pretwisting of a conjugated planar frame of the PSB5 molecule or whole retinal, by analogy to retinal in the rhodopsin protein pocket, can increase the photoisomerization quantum yield (for example, $72 \%$ for 18 trajectories of PSB ${ }^{16}$ ). In a representative trajectory of pretwisted PSB5, it was shown that hydrogen and carbon dihedrals around the active double bond synchronously reached the twisted CI geometry.

In the light of these observations, it might be interesting to examine (1) if PSB2 pretwisting can be used as a control knob for synchronous change of the $\mathrm{CN}_{2} \mathrm{C}_{3} \mathrm{C}$ and $\mathrm{HN}_{2} \mathrm{C}_{3} \mathrm{H}$ dihedrals during the molecule relaxation on the $S_{1}$ and (2) if the synchronous change of two dihedrals increases the quantum yield (trans product at $\mathrm{N}_{2} \mathrm{C}_{3}$ bond). 
To answer the first question, we prepare a thermal ensemble of PSB2 molecules with a pretwisted conjugated planar frame and track their photoreaction outcome. We simultaneously pretwist the thermalized molecule (strong harmonic restraint, as described in methods) along the three dihedrals, $\mathrm{C}_{1} \mathrm{~N}_{2} \mathrm{C}_{3} \mathrm{C}_{4}$ $\left(\gamma_{1}\right), \mathrm{N}_{2} \mathrm{C}_{3} \mathrm{C}_{4} \mathrm{C}_{5}\left(\gamma_{2}\right)$, and $\mathrm{C}_{3} \mathrm{C}_{4} \mathrm{C}_{5} \mathrm{C}_{6}\left(\gamma_{3}\right)$ to the values of analogous dihedral angles (listed in Table 2) surrounding the active bond of retinal in rhodopsin. ${ }^{16}$ As shown in Table 2 , in this pretwisted thermal ensemble 1, the fraction of synchronous trajectories (see Figure 4d) is $6.06 \%$, whereas in the unconstrained thermal ensemble (FSH) this fraction is $7.63 \%$. Therefore, these ensembles have a similar fraction of synchronous trajectories.

We can preselect certain properties in the new "generation" of trajectories (offsprings) by analogy to natural evolution and other optimal control methods. ${ }^{40}$ We generate new ensembles in which several PSB2 dihedral angles are restrained to values observed in molecular geometries in the unrestrained ensemble, which exhibited synchronized change of $\mathrm{CN}_{2} \mathrm{C}_{3} \mathrm{C}$ and $\mathrm{HN}_{2} \mathrm{C}_{3} \mathrm{H}$. We extract $\gamma_{1-3}$ from several synchronous trajectories and generate new ground-state thermal ensembles with these constrained $\gamma_{1-3}$ values (ensembles 2,3 , and $3 \mathrm{~b}$ in Table 2). In Table 2, we show that for these offspring ensembles the fraction of synchronous trajectories can increase up to $18.09 \%$.

In Table 3, we present the reaction outcomes for the ensemble 2. These results can help us to answer the second

Table 3. Reaction Outcomes for the Ensemble 2, Evaluated from 193 and 187 Trajectories using DSH and FSH Algorithms, Respectively

$\begin{array}{cccc}\text { outcome } & \mathrm{N}_{2} \mathrm{C}_{3} \text { cis } & \mathrm{N}_{2} \mathrm{C}_{3} \text { trans } & \mathrm{C}_{4} \mathrm{C}_{5} \\ P_{i, \mathrm{DSH}}[\%] & 39.9 & 46.1 & 14.0 \\ P_{i, \mathrm{FSH}}[\%] & 47.8 & 39.9 & 12.4\end{array}$

question. In particular, they show that the number of $\mathrm{N}_{2} \mathrm{C}_{3}$ trans outcomes increases from $34.8 \%$ (DSH) and 35.5\% (FSH) in the unrestrained thermal ensemble (Table 1) to $46.1 \%$ and $39.9 \%$ in the ensemble 2 . Therefore, synchronous motion of the dihedrals surrounding the active double bond increases the trans photoreaction outcome on the $\mathrm{N}_{2} \mathrm{C}_{3}$ bond.

In summary, we found that pretwisting of the initial molecular geometry of the photoexcited PSB2 molecule along several normal modes (dihedral angles) can significantly enhance the probability of the $\mathrm{C}_{4} \mathrm{C}_{5}$ reaction pathway. In addition, pretwisting of the PSB2 along several dihedral angles forming its planar conjugated frame can enhance its cis $\rightarrow$ trans isomerization around the $\mathrm{N}_{2} \mathrm{C}_{3}$ bond, as a result of synchronized $S_{1}$ PES dynamics of the two dihedral angles surrounding the rotating double bond. In practical applications, the required restraints on the initial chromophore structure may be realized by embedding the chromophore in an environment in which intermolecular interactions impose the required restraint or by introducing chemical modifications that impose intramolecular restrains. ${ }^{41}$ The above control mechanisms of PSB photoisomerization can have natural counterparts and can also be useful in various applications relying on ultrafast photoinduced reactions.

\section{ASSOCIATED CONTENT}

\section{(5) Supporting Information}

Methods, CAS molecular orbitals for the $S_{1}$ state of a representative initial geometry, $\operatorname{CASSCF}(4,4) / 6-31 G^{*}$ and
xMCQDPT2/CASSCF $(4,4) /$ cc-pVTZ energy profiles of $S_{0}$, $S_{1}$, and $S_{2}$ electronic states in PSB2 along the interpolated photoreaction pathways, $\operatorname{CASSCF}(4,4) / 6-31 \mathrm{G}^{*}$ energy profiles for rigid rotation of the $\mathrm{CN}_{2} \mathrm{C}_{3} \mathrm{C}$ and $\mathrm{CC}_{4} \mathrm{C}_{5} \mathrm{C}$ dihedrals in PSB2, description of the diabatic surface hopping method, approximation for expressing atomic displacements along normal modes in internal coordinates, energies of the PSB2 molecules displaced along the modes 1,2 , and 5, comparison of predicted energies and calculated energies of several PSB2 geometries, when multiple normal modes are excited, PSB2 geometry at the $S_{0}$ minimum, references, animation of atomic displacements along the mode 5 in PSB2. This material is available free of charge via the Internet at http://pubs.acs.org.

\section{AUTHOR INFORMATION}

\section{Corresponding Author}

*E-mail: Lvukov1@gmail.com (L.V.); gerrit.x.groenhof@jyu.fi (G.G.).

\section{Notes}

The authors declare no competing financial interest.

\section{ACKNOWLEDGMENTS}

G.G. thanks the Academy of Finland for financial support. L.V. gratefully acknowledges the support from the DAAD research grant. We acknowledge Maarten Wolf, Timo M. Graen, Helmut Grubmüller, and Dmitry Morozov for useful discussions.

\section{REFERENCES}

(1) Zechmeister, L. Cis-trans Isomerization and Stereochemistry of Carotenoids and Diphenyl-polyenes. Chem. Rev. 1944, 34, 267-344.

(2) Hugel, T.; Holland, N. B.; Cattani, A.; Moroder, L.; Seitz, M.; Gaub, H. E. Single-Molecule Optomechanical Cycle. Science 2002, 296, 1103-1106.

(3) Koumura, N.; Zijlstra, R. W. J.; van Delden, R. A.; Harada, N.; Feringa, B. L. Light-Driven Monodirectional Molecular Rotor. Nature 1999, 401, 152-155.

(4) Palczewski, K. G Protein-Coupled Receptor Rhodopsin. Annu. Rev. Biochem. 2006, 75, 743-767.

(5) Zhang, F.; Prigge, M.; Beyriere, F.; Tsunoda, S. P.; Mattis, J.; Yizhar, O.; Hegemann, P.; Deisseroth, K. Red-Shifted Optogenetic Excitation: A Tool for Fast Neural Control Derived from Volvox carteri. Nat. Neurosci. 2008, 11, 631-633.

(6) Gunaydin, L. A.; Yizhar, O.; Berndt, A.; Sohal, V. S.; Deisseroth, K.; Hegemann, P. Ultrafast Optogenetic Control. Nat. Neurosci. 2010, 13, 387-392.

(7) Warshel, A. Bicycle-Pedal Model for the First Step in the Vision Process. Nature 1976, 260, 679-683.

(8) Wang, Q.; Schoenlein, R.; Peteanu, L.; Mathies, R.; Shank, C. Vibrationally Coherent Photochemistry in the Femtosecond Primary Event of Vision. Science 1994, 266, 422-424.

(9) Polli, D.; Altoè, P.; Weingart, O.; Spillane, K. M.; Manzoni, C.; Brida, D.; Tomasello, G.; Orlandi, G.; Kukura, P.; Mathies, R. A.; Garavelli, M.; Cerullo, G. Conical Intersection Dynamics of the Primary Photoisomerization Event in Vision. Nature 2010, 467, 440443.

(10) Weingart, O.; Schapiro, I.; Buss, V. Bond Torsion Affects the Product Distribution in the Photoreaction of Retinal Model Chromophores. J. Mol. Model. 2006, 12, 713-721.

(11) Frutos, L. M.; Andruniów, T.; Santoro, F.; Ferré, N.; Olivucci, M. Tracking the Excited-state Time Evolution of the Visual Pigment with Multiconfigurational Quantum Chemistry. Proc. Natl. Acad. Sci. U.S.A. 2007, 104, 7764-7769.

(12) Andruniów, T.; Ferré, N.; Olivucci, M. Structure, Initial ExcitedState Relaxation, and Energy Storage of Rhodopsin Resolved at the Multiconfigurational Perturbation Theory Level. Proc. Natl. Acad. Sci. U.S.A. 2004, 101, 17908-17913. 
(13) Bonačić-Koutecký, V.; Köhler, J.; Michl, J. Prediction of Structural and Environmental Effects on the $S_{1}-S_{0}$ Energy Gap and Jump Probability in Double-Bond cis-trans Photoisomerization. Chem. Phys. Lett. 1984, 104, 440-443.

(14) Freedman, K. A.; Becker, R. S. Comparative Investigation of the Photoisomerization of the Protonated and Unprotonated $n$-Butylamine Schiff Bases of 9-cis-, 11-cis-, 13-cis-, and all-trans-Retinals. J. Am. Chem. Soc. 1986, 108, 1245-1251.

(15) Ben-Nun, M.; Molnar, F.; Lu, H.; Phillips, J. C.; Martínez, T. J.; Schulten, K. Quantum Dynamics of the Femtosecond Photoisomerization of Retinal in Bacteriorhodopsin. Faraday Discuss. 1998, 110, 447-462.

(16) Weingart, $\mathrm{O}$. The Twisted $\mathrm{C} 11=\mathrm{C} 12$ Bond of the Rhodopsin ChromophoresA Photochemical Hot Spot. J. Am. Chem. Soc. 2007, 129, 10618-10619.

(17) Szymczak, J. J.; Barbatti, M.; Lischka, H. Mechanism of Ultrafast Photodecay in Restricted Motions in Protonated Schiff Bases: The Pentadieniminium Cation. J. Chem. Theory Comput. 2008, 4, 11891199.

(18) Szymczak, J. J.; Barbatti, M.; Lischka, H. Is the Photoinduced Isomerization in Retinal Protonated Schiff Bases a Single- or DoubleTorsional Process. J. Phys. Chem. A 2009, 113, 11907-11918.

(19) Sinicropi, A.; Migani, A.; De Vico, L.; Olivucci, M. Photoisomerization Acceleration in Retinal Protonated Schiff-Base Models. Photochem. Photobiol. Sci. 2003, 2, 1250-1255.

(20) Logunov, S. L.; Song, L.; El-Sayed, M. A. Excited-State Dynamics of a Protonated Retinal Schiff Base in Solution. J. Phys. Chem. 1996, 100, 18586-18591.

(21) Cembran, A.; Bernardi, F.; Olivucci, M.; Garavelli, M. The Retinal Chromophore/Chloride Ion Pair: Structure of the Photoisomerization Path and Interplay of Charge Transfer and Covalent States. Proc. Natl. Acad. Sci. U.S.A. 2005, 102, 6255-6260.

(22) Sovdat, T.; Bassolino, G.; Liebel, M.; Schnedermann, C.; Fletcher, S. P.; Kukura, P. Backbone Modification of Retinal Induces Protein-like Excited State Dynamics in Solution. J. Am. Chem. Soc. 2012, 134, 8318-8320.

(23) Röhrig, U. F.; Guidoni, L.; Laio, A.; Frank, I.; Rothlisberger, U. A Molecular Spring for Vision. J. Am. Chem. Soc. 2004, 126, 1532815329.

(24) Ben-Nun, M.; Molnar, F.; Schulten, K.; Martínez, T. J. The Role of Intersection Topography in Bond Selectivity of cis-trans Photoisomerization. Proc. Natl. Acad. Sci. U.S.A. 2002, 99, 1769-1773.

(25) Lin, S. W.; Groesbeek, M.; van der Hoef, I.; Verdegem, P.; Lugtenburg, J.; Mathies, R. A. Vibrational Assignment of Torsional Normal Modes of Rhodopsin: Probing Excited-State Isomerization Dynamics along the Reactive $\mathrm{C}_{11}=\mathrm{C}_{12}$ Torsion Coordinate. J. Phys. Chem. B 1998, 102, 2787-2806.

(26) Hayashi, S.; Tajkhorshid, E.; Schulten, K. Photochemical Reaction Dynamics of the Primary Event of Vision Studied by Means of a Hybrid Molecular Simulation. Biophys. J. 2009, 96, 403-416.

(27) Prokhorenko, V. I.; Nagy, A. M.; Waschuk, S. A.; Brown, L. S.; Birge, R. R.; Miller, R. J. D. Coherent Control of Retinal Isomerization in Bacteriorhodopsin. Science 2006, 313, 1257-1261.

(28) Garavelli, M.; Bernardi, F.; Celani, P.; Robb, M. A.; Olivucci, M. Minimum Energy Paths in the Excited and Ground States of Short Protonated Schiff Bases and of the Analogous Polyenes. J. Photochem. Photobiol. A 1998, 114, 109-116.

(29) Garavelli, M.; Bernardi, F.; Robb, M. A.; Olivucci, M. The ShortChain Acroleiniminium and Pentadieniminium Cations: Towards a Model for Retinal Photoisomerization. A CASSCF/PT2 Study. J. Mol. Struct.: THEOCHEM 1999, 463, 59-64.

(30) Fabiano, E.; Groenhof, G.; Thiel, W. Approximate Switching Algorithms for Trajectory Surface Hopping. Chem. Phys. 2008, 351, $111-116$.

(31) Tully, J. Molecular Dynamics with Electronic Transitions. J. Chem. Phys. 1990, 93, 1061-1071.

(32) Granovsky, A. A. Extended Multi-Configuration QuasiDegenerate Perturbation Theory: The New Approach to Multi-State
Multi-Reference Perturbation Theory. J. Chem. Phys. 2011, 134, 214113-14

(33) Gozem, S.; Huntress, M.; Schapiro, I.; Lindh, R.; Granovsky, A. A.; Angeli, C.; Olivucci, M. Dynamic Electron Correlation Effects on the Ground State Potential Energy Surface of a Retinal Chromophore Model. J. Chem. Theory Comput. 2012, 8, 4069-4080.

(34) Volland, W. V.; Davidson, E. R.; Borden, W. T. Effect of Carbon Atom Pyramidalization on the Bonding in Ethylene. J. Am. Chem. Soc. 1979, 101, 533-537.

(35) Weingart, O. The Role of HOOP-Modes in the Ultrafast PhotoIsomerization of Retinal Models. Chem. Phys. 2008, 349, 348-355.

(36) Weingart, O.; Altoè, P.; Stenta, M.; Bottoni, A.; Orlandi, G.; Garavelli, M. Product Formation in Rhodopsin by Fast Hydrogen Motions. Phys. Chem. Chem. Phys. 2011, 13, 3645-3648.

(37) Schapiro, I.; Ryazantsev, M. N.; Frutos, L. M.; Ferré, N.; Lindh, R; Olivucci, M. The Ultrafast Photoisomerizations of Rhodopsin and Bathorhodopsin Are Modulated by Bond Length Alternation and HOOP Driven Electronic Effects. J. Am. Chem. Soc. 2011, 133, 33543364.

(38) Boggio-Pasqua, M.; Burmeister, C. F.; Robb, M. A.; Groenhof, G. Photochemical Reactions in Biological Systems: Probing the Effect of the Environment by Means of Hybrid Quantum Chemistry/ Molecular Mechanics Simulations. Phys. Chem. Chem. Phys. 2012, 14, $7912-7928$.

(39) Klaffki, N.; Weingart, O.; Garavelli, M.; Spohr, E. Sampling Excited State Dynamics: Influence of HOOP Mode Excitations in a Retinal Model. Phys. Chem. Chem. Phys. 2012, 14, 14299-14305.

(40) Judson, R. S.; Rabitz, H. Teaching Lasers to Control Molecules. Phys. Rev. Lett. 1992, 68, 1500-1503.

(41) Kandori, H.; Sasabe, H.; Nakanishi, K.; Yoshizawa, T.; Mizukami, T.; Shichida, Y. Real-Time Detection of 60-fs Isomerization in a Rhodopsin Analog Containing Eight-Membered-Ring Retinal. J. Am. Chem. Soc. 1996, 118, 1002-1005. 